\title{
Dimethachlon Resistance in Sclerotinia sclerotiorum in China
}

Feng Zhou, Xiao-Lei Zhang, Jin-Li Li, and Fu-Xing Zhu, College of Plant Science and Technology, Huazhong Agricultural University, Wuhan, 430070, China

\begin{abstract}
Zhou, F., Zhang, X.-L., Li, J.-L., and Zhu, F.-X. 2014. Dimethachlon resistance in Sclerotinia sclerotiorum in China. Plant Dis. 98:1221-1226.

The dicarboximide fungicide dimethachlon has been widely used for controlling Sclerotinia sclerotiorum in China for more than a decade. To assess the current status of dimethachlon resistance in S. sclerotiorum in China, 2,424 isolates were collected from disease-infected oilseed rape and soybean plants in five provinces of China in 2011 and 2012, and dimethachlon resistance was monitored by mycelial growth inhibition method on potato dextrose agar (PDA) media. Dimethachlon at $5 \mu \mathrm{g} / \mathrm{ml}$ was used as a discriminatory dose to detect resistance in all isolates, and $50 \%$ effective concentration values were determined for all dimethachlon-resistant isolates and some sensitive isolates. No dimethachlon resistance was detected in isolates from Anhui province (eastern China), Gansu province (northwestern China), and Qinghai province (western China). In Hunan province (central China), 3 of 268 $(1.12 \%)$ isolates collected from oilseed rape plants in 2012 were re-

sistant to dimethachlon, and the resistance ratios for the three resistant isolates were $4.56,32.70$, and 105.53 , respectively. In Heilongjiang province (northeastern China), 8 of 243 (3.29\%) isolates collected from soybean plants in 2011 were resistant to dimethachlon, with resistance ratios of 5.57 to $94.80 ; 11$ of 409 (2.69\%) isolates collected in 2012 were resistant to dimethachlon, with resistance ratios of 3.21 to 9.69. Cross-resistance studies showed that there was positive crossresistance between dimethachlon and iprodione, procymidone, and the $\mathrm{N}$-phenyl carbamate fungicide diethofencarb. No cross-resistance was found between dimethachlon and carbendazim, tebuconazole, kresoxim-methyl, thiram, and boscalid. Compared with the sensitive isolates of S. sclerotiorum, the field-dimethachlon-resistant isolates were more sensitive to osmotic pressure, grew more slowly on PDA media, and were less pathogenic on leaves of oilseed rape.
\end{abstract}

Sclerotinia sclerotiorum (Lib.) de Bary is one of the most devastating and economically important necrotrophic fungal phytopathogens. It is capable of infecting more than 400 species of plants worldwide (3), including numerous weeds and important crops such as soybean, sunflower, canola, and oilseed rape (28). Infections of oilseed rape plants by germinating ascospores of $S$. sclerotiorum occur initially on flowers or petals in spring. When the infected petals fall, many will adhere to leaves, stems, and early pods $(1,7,33)$. Sclerotinia stem rot caused by $S$. sclerotiorum is severe in many countries, including Canada (2), the United States (4), Australia (19), and China (22,30,38). In China alone, the annual oilseed rape acreage is more than 7 million ha (34). Annual oilseed rape yield losses caused by $S$. sclerotiorum range from 5 to $30 \%$ and yield losses of severely infected fields are as high as $80 \%$ (12). Because breeding programs for disease-resistant oilseed rape have been hampered by limited genetic resources (21), S. sclerotiorum continues to impose serious limitations on oilseed rape production worldwide (35).

Application of fungicides is an important tool for controlling $S$. sclerotiorum (22). The benzimidazole fungicide carbendazim has been widely and extensively used against $S$. sclerotiorum for several decades in China $(30,39)$. High levels of carbendazim resistance and reduced field efficacy have been reported $(17,27,29,36,37)$. Widespread control failures due to carbendazim resistance resulted in a suspension of carbendazim application for control of $S$. sclerotiorum in the 2000 s in eastern China, especially in Jiangsu province $(27,30)$. Dimethachlon, a dicarboximide fungicide, has been widely used to control Sclerotinia stem rot in China for many years. In Jiangsu province, dimethachlon has been recommended to supersede carbendazim for the management of Sclerotinia stem rot since 2002 (17). Wide use of dimethachlon and other dicarboximide fungicides has raised concerns about re-

Corresponding author: F.-X. Zhu, E-mail: zhufuxing@mail.hzau.edu.cn

Accepted for publication 11 March 2014.

http://dx.doi.org/10.1094/PDIS-10-13-1072-RE

(C) 2014 The American Phytopathological Society sistance issues in $S$. sclerotiorum. Furthermore, dimethachloninsensitive isolates of $S$. sclerotiorum have been detected in oilseed rape fields in Jiangsu province (22). A previous study demonstrated that a high level of dimethachlon resistance in S. sclerotiorum could be induced by consecutive selection on dimethachlonamended potato dextrose agar (PDA) media in a laboratory setting (22). Although a high level of resistance to dicarboximide fungicides has not yet been reported in field isolates of $S$. sclerotiorum, resistance to the dicarboximide fungicide iprodione has been detected in closely related plant pathogens such as $S$. homoeocarpa (15), S. minor (14), Botrytis cinerea (24), and Monilinia fructicola (9). Therefore, it is necessary to monitor dimethachlon resistance and to study the biological characteristics of field-resistant isolates of $S$. sclerotiorum once resistance has emerged. The objectives of this study were to (i) monitor dimethachlon resistance in S. sclerotiorum isolates collected from different provinces of China; (ii) explore cross-resistance patterns between dimethachlon and other widely used fungicides, including iprodione, procymidone, fludioxonil, carbendazim, diethofencarb, tebuconazole, kresoxim-methyl, thiram, and boscalid; and (iii) compare biological characteristics such as mycelial growth rate, sclerotial production on PDA, pathogenicity on host plants, and osmotic sensitivity between the dimethachlon-resistant isolates and the sensitive isolates.

\section{Materials and Methods}

Isolates of S. sclerotiorum. Isolates of S. sclerotiorum were collected from oilseed rape plants with symptoms of Sclerotinia stem rot in central China (Hunan province), eastern China (Anhui province), western China (Qinghai province), and northwestern China (Gansu province) and from diseased soybean plants in northeastern China (Heilongjiang province) in 2011 and 2012 (Fig. 1). In Anhui, Hunan, Heilongjiang, and Qinghai provinces, isolates were collected from more than 20 fields per province. In Gansu province, isolates were collected from 15 fields. The fields were separated from each other by more than $20 \mathrm{~km}$. In each field, sclerotia were randomly collected from crop plants with symptoms of Sclerotinia stem rot. Sclerotia collected from the same diseased plant were regarded as one isolate. One dimethachlon-sensitive isolate (SXMG) and one dimethachlon-resistant isolate (SX2) col- 
lected from Shaanxi province were also used in this study for comparison.

Fungicides. All fungicides used in this study were technicalgrade products. Dimethachlon $(96.2 \%)$ was provided by Wenzhou Pesticide Co. Ltd. and dissolved in methanol to produce a 1,000$\mu \mathrm{g} / \mathrm{ml}$ stock solution. Carbendazim $(98.1 \%)$ was provided by Haili Guixi Chemical Co. Ltd. and dissolved in hydrochloric acid $(\mathrm{HCl})$ at $0.1 \mathrm{~mol} / \mathrm{liter}$ to produce a $1,000-\mu \mathrm{g} / \mathrm{ml}$ stock solution. Iprodione (95.3\%) and procymidone $(98.0 \%)$ were provided by Heyi Agrochemical Co. Ltd. Tebuconazole (96.2\%) was provided by Sheyang Huanghai Pesticide Chemical Co. Ltd. Diethofencarb (95.4\%) was provided by Lanfeng Chemical Company. Kresoxim-methyl (96.6\%) was provided by Gengyun Chemical Co. Ltd. Thiram (96.0\%) was provided by Guanlong Agrochemical Co. Ltd. Boscalid $(97.0 \%)$ was provided by Kangbaotai Fine-Chemical Co. Ltd. All fungicides, except for dimethachlon and carbendazim, were dissolved in acetone to produce $1,000-\mu \mathrm{g} / \mathrm{ml}$ stock solutions.

Resistance determination. Resistance determination was performed according to Ma et al. (22), with a few modifications. Sclerotia of S. sclerotiorum were bisected, sterilized in $75 \%$ alcohol for $3 \mathrm{~min}$, transferred into $1 \%$ sodium hypochlorite solution, sterilized for $3 \mathrm{~min}$, rinsed in sterile distilled water for $30 \mathrm{~s}$, and dried on sterilized filter paper. Halves of sclerotia were placed on PDA (200 $\mathrm{g}$ of potato, $20 \mathrm{~g}$ of agar, and $20 \mathrm{~g}$ of dextrose per liter of distilled water) plates. Plates were incubated for 2 days at $26^{\circ} \mathrm{C}$ in a growth chamber with a $12-\mathrm{h}$ photoperiod. Dimethachlon at $5 \mu \mathrm{g} / \mathrm{ml}$ was used as the discriminatory dose. Mycelial plugs $(6 \mathrm{~mm}$ in diameter) cut from the fresh margins of 48-h-old colonies were transferred upside down onto PDA amended with dimethachlon at 5 $\mu \mathrm{g} / \mathrm{ml}$, using fungicide-free PDA as the control. After $48 \mathrm{~h}$ of incubation at $26^{\circ} \mathrm{C}$ in a growth chamber, mycelial growth was measured in two perpendicular directions. Isolates that showed growth on dimethachlon at $5 \mu \mathrm{g} / \mathrm{ml}$ were tentatively considered resistant, whereas the completely inhibited isolates were considered sensitive to dimethachlon (22). The percentage of resistant isolates was calculated as follows: percentage of resistant isolates $(\%)=$ total number of resistant isolates/total number of isolates detected $\times 100$.

For all of the tentatively identified resistant isolates and some arbitrarily selected sensitive isolates, half maximal (50\%) effective concentrations $\left(\mathrm{EC}_{50}\right)$ were determined as follows. PDA media amended with dimethachlon at $0,1,2,4,8,16,32,64$, and 128 $\mu \mathrm{g} / \mathrm{ml}$ were used for all resistant isolates. PDA media amended with dimethachlon at $0,0.125,0.25,0.5,1.0$, and $2.0 \mu \mathrm{g} / \mathrm{ml}$ were used for sensitive isolates. For cross-resistance determination, at least five concentrations of each fungicide were used for $\mathrm{EC}_{50}$

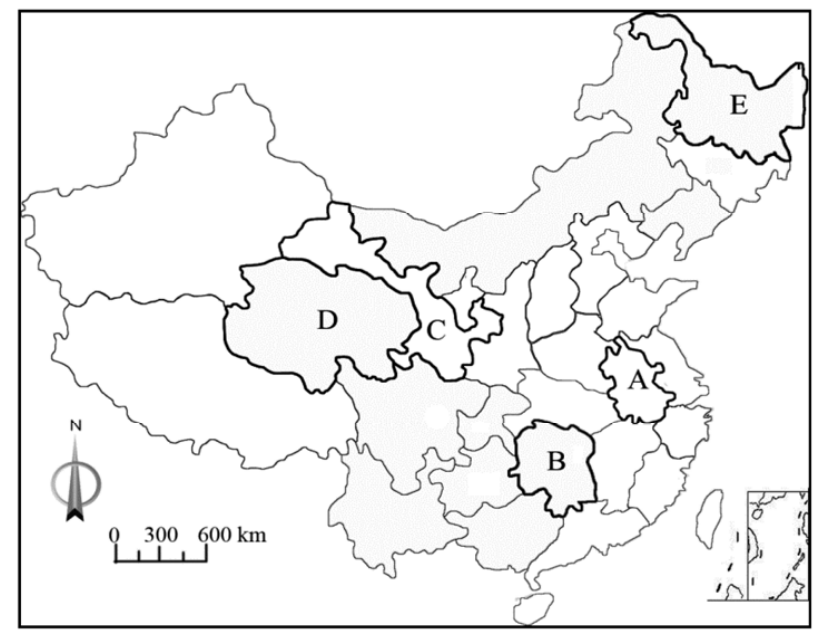

Fig. 1. Map of China. Letters A, B, C, and D represent Anhui, Hunan, Gansu, and Qinghai provinces, respectively, where isolates of Sclerotinia sclerotiorum were collected from oilseed rape plants with symptoms of Sclerotinia stem rot. Letter $\mathrm{E}$ represents Heilongjiang province, where isolates of $S$. sclerotiorum were collected from soybean plants. assays. There were three replicates for each concentration and all tests were performed twice. Resistance ratio for the resistant isolate was calculated as follows: resistance ratio $=\mathrm{EC}_{50}$ value of resistant isolate/average $\mathrm{EC}_{50}$ value of sensitive isolates.

The average $\mathrm{EC}_{50}$ value of sensitive isolates was determined from 214 isolates sampled from the five provinces.

Osmotic sensitivity determination. Three field-dimethachlonsensitive isolates (SXMG, GUMG, and HLJMG) and three fielddimethachlon-resistant isolates (HN1, HLJ4, and SX2) were used to determine osmotic sensitivity. For each isolate, mycelial plugs (6 $\mathrm{mm}$ in diameter) were taken from the margins of 48-h-old colonies and transferred onto PDA media amended with $\mathrm{NaCl}$ (at $0,1.25$, $2.5,5.0,10,20,40$, and $80 \mathrm{~g} /$ liter) or glucose (at $0,10,20,40$, $80,100,150$, and $200 \mathrm{~g} /$ liter). PDA free of $\mathrm{NaCl}$ and glucose was used as the untreated control. Six plates (replicates) for each concentration of $\mathrm{NaCl}$ and glucose were incubated at $26^{\circ} \mathrm{C}$ for $48 \mathrm{~h}$ in a growth chamber with a 12 -h photoperiod. Diameters of fungal colonies were measured perpendicularly in millimeters. Percentage of mycelial radial growth inhibition relative to the $\mathrm{NaCl}$ - and glucose-free control was calculated. The experiment was performed twice.

Characterization of sclerotial production and mycelial growth on PDA. The same three sensitive and three resistant isolates used in the osmotic sensitivity experiment were characterized for their sclerotial production and mycelial growth on PDA media. For each isolate, mycelial plugs $(6 \mathrm{~mm}$ in diameter $)$ was taken from the margins of 48-h-old colonies and transferred onto PDA plates. Six plates for each isolate were incubated at $26^{\circ} \mathrm{C}$ for 21 days in a growth chamber with a 12-h photoperiod. The plates were observed every day. After $48 \mathrm{~h}$ of incubation, the diameter of each fungal colony was measured perpendicularly in millimeters. The start time for sclerotia formation (the number of days from incubation to initial formation of sclerotia) and the end time for sclerotia growth (the number of days from incubation to the end of growth of sclerotia) on PDA plates were recorded. At the end of 21 days of incubation, the number and distribution of sclerotia on PDA plates were recorded. All sclerotia in each dish were weighed after drying at $50^{\circ} \mathrm{C}$ for 2 days.

Determination of pathogenicity on detached leaves of host plants. Three field-dimethachlon-resistant isolates and four sensitive isolates were used for pathogenicity tests on oilseed rape plants. Leaves of similar growth position and size were detached from oilseed rape plants, rinsed with sterile water, air dried in a transfer hood, transferred to $15-\mathrm{cm}$ petri dishes lined with wet filter paper to maintain high humidity, and then inoculated on the adaxial surface with mycelial plugs $(6 \mathrm{~mm}$ in diameter) cut from the margins of 48 -h-old colonies of $S$. sclerotiorum isolates. A mycelial plug of a sensitive isolate and another plug of a resistant isolate were placed on opposite sides of the midrib. The inoculated leaves were incubated in a growth chamber $\left(26^{\circ} \mathrm{C}\right.$, photoperiod of $\left.16 \mathrm{~h}\right)$. Lesions were measured after 24,48 , and $72 \mathrm{~h}$ of incubation. The experiment was performed twice, with at least six replicates for each isolate.

Data analysis. $\mathrm{EC}_{50}$ values were calculated by the Bioassay Analysis for Quantity Data procedure in Data Processing System (ver. 7.05; Hangzhou RuiFeng Information Technology Co. Ltd.). The analysis of variance procedure in Statistical Product and Service Solutions (ver. 17.0; SPSS Inc.) and Fisher's least significant difference test $(P=0.05)$ were used to test for significant differences in biological characteristics such as mycelial growth rate, sclerotial production, pathogenicity, and osmotic sensitivity among different isolates. Graphs were created in Microsoft Excel (ver. 2010; Microsoft Corporation).

\section{Results}

Dimethachlon resistance in field isolates of $S$. sclerotiorum in China. Dimethachlon resistance was investigated in a total of 2,424 field isolates of S. sclerotiorum collected in 2011 and 2012. Only 22 isolates were resistant to dimethachlon, and the overall resistance frequency (percentage of resistant isolates) was $0.91 \%$, 
indicating that the development of dimethachlon resistance in $S$. sclerotiorum was still at the initial stage (Table 1). Of the five provinces where field isolates were collected, dimethachlon-resistant isolates were detected only from Hunan province and Heilongjiang province. One isolate with a resistance ratio as high as 105.53 was collected from Hunan province.

Spectrum of cross-resistance in field-resistant isolates. There was positive cross-resistance among the dicarboximide fungicides dimethachlon, iprodione, and procymidone, which share the same mode of action (Table 2). Positive cross-resistance was also found between the dicarboximide fungicides and the carbamate fungicide diethofencarb, whose mode of action is different from that of the dicarboximide fungicides. The target of diethofencarb is $\beta$-tubulin assembly in mitosis, whereas the target of the dicarboximide fungicides is mitogen-activated protein (MAP)/histidine kinase in osmotic signal transduction. No cross-resistance was found between the dicarboximide fungicides and other fungicides such as carbendazim, tebuconazole, kresoxim-methyl, boscalid, and thiram.

Table 1. Dimethachlon resistance in field isolates of Sclerotinia sclerotiorum in five provinces of China ${ }^{\mathrm{v}}$

\begin{tabular}{|c|c|c|c|c|c|c|}
\hline \multirow[b]{2}{*}{ Province } & \multirow[b]{2}{*}{ Host } & \multirow[b]{2}{*}{ Year } & \multirow[b]{2}{*}{ Monitored $(n)^{\mathrm{w}}$} & \multirow[b]{2}{*}{ Resistant $(\%)^{x}$} & \multicolumn{2}{|c|}{ Median (minimum-maximum) } \\
\hline & & & & & $\mathrm{EC}_{50}(\mu \mathrm{g} / \mathrm{ml})^{\mathrm{y}}$ & Resistance ratio $^{\mathrm{z}}$ \\
\hline \multirow[t]{2}{*}{ Hunan } & Oilseed rape & 2011 & 151 & 0 & - & - \\
\hline & & 2012 & 268 & $3(1.12)$ & $9.69(1.35-31.27)$ & $32.70(4.56-105.53)$ \\
\hline \multirow[t]{2}{*}{ Anhui } & Oilseed rape & 2011 & 227 & 0 & - & - \\
\hline & & 2012 & 336 & 0 & - & - \\
\hline \multirow[t]{2}{*}{ Qinghai } & Oilseed rape & 2011 & 314 & 0 & - & - \\
\hline & & 2012 & - & - & - & - \\
\hline \multirow[t]{2}{*}{ Gansu } & Oilseed rape & 2011 & 55 & 0 & - & - \\
\hline & & 2012 & 421 & 0 & - & - \\
\hline \multirow{2}{*}{ Heilongjiang } & Soybean & 2011 & 243 & $8(3.29)$ & $9.96(1.65-28.09)$ & 33.61 (5.57-94.80) \\
\hline & & 2012 & 409 & $11(2.69)$ & $1.69(0.95-2.87)$ & $5.70(3.21-9.69)$ \\
\hline
\end{tabular}

v Symbol: - indicates no data available or could not be calculated.

${ }^{\mathrm{w}}$ Number of isolates monitored.

${ }^{\mathrm{x}}$ Number and frequency $(\%)$ of resistant isolates. Resistance frequency $(\%)=$ number of resistant isolates/number of isolates monitored $\times 100$.

${ }^{y}$ Half maximal $(50 \%)$ effective concentrations $\left(\mathrm{EC}_{50}\right)$ values of resistant isolates.

${ }^{\mathrm{z}} \mathrm{EC}_{50}$ value of resistant isolate/average $\mathrm{EC}_{50}$ value of sensitive isolates. The average $\mathrm{EC}_{50}$ value of sensitive isolates was $0.2963 \mu \mathrm{g} / \mathrm{ml}$.

Table 2. Cross-resistance to different fungicides in field-resistant isolates of Sclerotinia sclerotiorum

\begin{tabular}{|c|c|c|c|c|c|c|c|}
\hline & & \multicolumn{6}{|c|}{ Dimethachlon $\mathrm{EC}_{50}$ values $(\mu \mathrm{g} / \mathrm{ml})^{\mathrm{z}}$} \\
\hline \multicolumn{2}{|c|}{ Fungicides } & \multicolumn{3}{|c|}{ Dimethachlon-resistant isolates } & \multicolumn{3}{|c|}{ Field-sensitive isolates } \\
\hline Fungicide group & Common name & HN1 & HLJ4 & SX2 & SXMG & HLJMG & GSMG \\
\hline \multirow[t]{3}{*}{ Dicarboximide } & Dimethachlon & 31.27 & 28.10 & 29.57 & 0.38 & 0.22 & 0.23 \\
\hline & Iprodione & $>40$ & $>40$ & 26.28 & 0.15 & 0.26 & 0.13 \\
\hline & Procymidone & $>40$ & $>40$ & $>40$ & 0.23 & 0.21 & 0.23 \\
\hline Phenylpyrrole & Fludioxonil & 0.0012 & 1.08 & 0.0010 & 0.0012 & 0.0013 & 0.0013 \\
\hline Benzimidazole & Carbendazim & 0.06 & 0.99 & 0.06 & 0.07 & 0.05 & 0.07 \\
\hline Carbamate & Diethofencarb & 19.49 & 35.64 & 22.57 & 0.51 & 0.36 & 0.52 \\
\hline Triazole & Tebuconazole & 0.50 & 0.91 & 0.51 & 0.22 & 0.17 & 0.05 \\
\hline Strobilurin & Kresoxim-methyl & 0.90 & 0.92 & 0.76 & 0.29 & 0.07 & 0.15 \\
\hline Organo-sulfur & Thiram & 0.66 & 1.65 & 0.34 & 1.2 & 0.92 & 0.89 \\
\hline Pyridine-carboxamide & Boscalid & 0.11 & 0.09 & 0.22 & 0.06 & 0.02 & 0.06 \\
\hline
\end{tabular}

${ }^{\mathrm{z}} \mathrm{EC}_{50}=$ half maximal (50\%) effective concentrations of resistant isolates. Dimethachlon-resistant isolates HN1, HLJ4, and SX2 were collected from Hunan, Heilongjiang, and Shaanxi provinces, respectively. Field-sensitive isolates SXMG, HLJMG, and GSMG were collected from Shaanxi, Heilongjiang, and Gansu provinces, respectively.

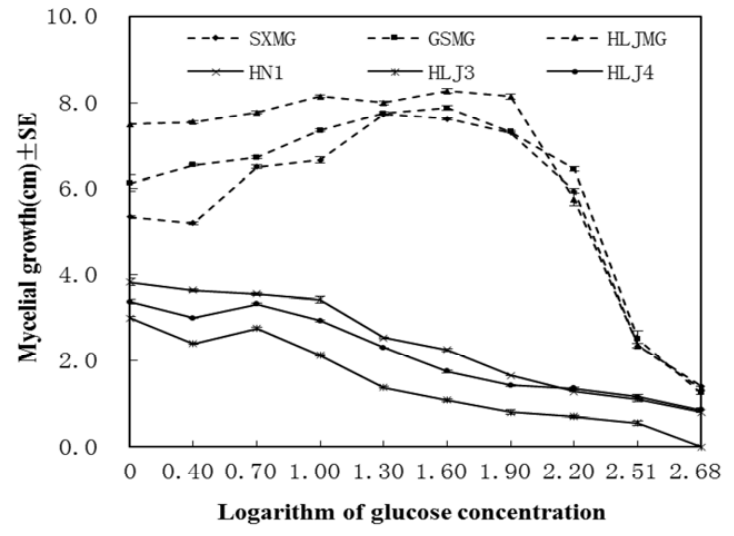

A. Sensitivity to glucose

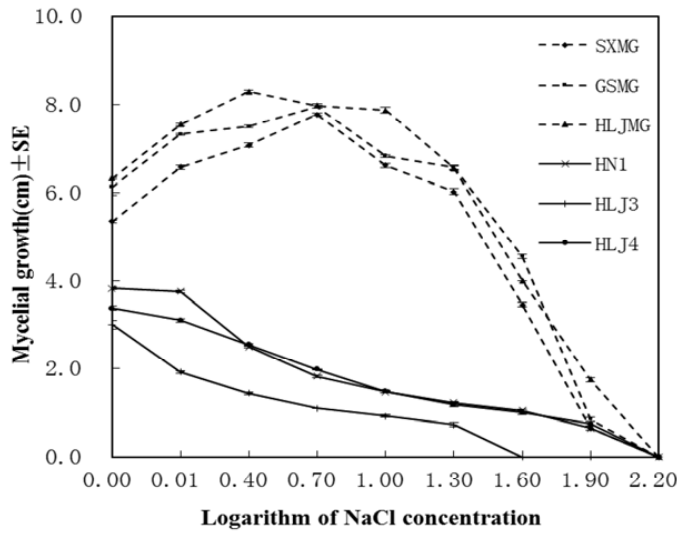

B. Sensitivity to $\mathrm{NaCl}$

Fig. 2. Comparison of osmotic sensitivity between sensitive isolates (SXMG, GSMG, and HIJMG) and dimethachlon-resistant isolates (HN1, HLJ3, and HLJ4). Isolates SXMG, GSMG, and HN1 were collected from Shaanxi, Gansu, and Hunan provinces, respectively. Isolates HIJMG, HLJ3, and HLJ4 were collected from Heilongjiang province. Data represent the means of mycelial growth inhibition \pm standard error (SE) of at least six replicates for each treatment. A, Sensitivity to glucose; B, sensitivity to $\mathrm{NaCl}$. 
Osmotic sensitivity. The field-dimethachlon-resistant isolates grew more slowly than field-sensitive isolates on PDA amended with $\mathrm{NaCl}$ (lower than $40 \mathrm{~g} / \mathrm{liter}$ ) or glucose (lower than 200 $\mathrm{g} / \mathrm{liter}$, indicating that the field-resistant isolates were more sensitive to osmotic pressure (Fig. 2).

Mycelial growth rate and sclerotial production on PDA. Compared with the field-sensitive isolates, there was significantly $(P<0.001)$ slower mycelial growth on PDA for the dimethachlonresistant isolates (Table 3). No definite conclusion could be drawn about differences in the amount and dry weight of sclerotia between the dimethachlon-resistant isolates and the sensitive isolates. It was obvious that the start time and end time for sclerotial production in all three of the resistant isolates was delayed compared with the sensitive isolates.

Pathogenicity on detached leaves of host plants. Pathogenicity of the field-dimethachlon-resistant isolates was significantly lower $(P<0.001)$ than that of the sensitive isolates (Table 4). Resistant isolates SX2 and HLJ3 completely lost pathogenicity on detached leaves of oilseed rape. Lesion diameters and disease incidences of the resistant isolates were much lower than those of the sensitive isolates, especially at $72 \mathrm{~h}$. Furthermore, the speed of mycelial invasion into plant tissue, as measured by lesion diameter at the first $24 \mathrm{~h}$, decreased obviously for the resistant isolates. Actually, no lesion symptoms were observable with the naked eye after the first $24 \mathrm{~h}$ for the resistant isolates, whereas lesion symptoms were apparent for the sensitive isolates.

\section{Discussion}

Field isolates of $S$. sclerotiorum with reduced sensitivity to dimethachlon were detected in Jiangsu province in 2006 and 2007 (22). Medium to high levels of resistance to dimethachlon have been detected in our laboratory in samples from Shaanxi province (data not shown). In the adjacent Gansu province, no resistant isolate was detected. The geographic distribution of resistant isolates could not be explained by the intensity of dimethachlon application. The benzimidazole fungicide carbendazim and the dicarboximide fungicides dimethachlon, procymidone, and iprodione; the triazole fungicide tebuconazole; and the imidazole fungicide prochloraz were used to control Sclerotinia rot in oilseed rape and soybean fields in China. Dimethachlon has been one of the major fungicides in controlling Sclerotinia stem rot in oilseed rape after carbendazim resistance became widespread in eastern China $(22,30)$. However, no resistant isolate was found in Anhui province. In Hunan province and Heilongjiang province, where use of dimethachlon was not as extensive as in eastern China (20), dimethachlon resistance was detected and some isolates even evolved high resistance to dimethachlon. In general, dimethachlon resistance frequencies were still low in China and the resistance seemed to be still at the "emergence phase" stage of the development of fungicide resistance (5). High resistance levels with resistance ratios greater than 50 for some isolates from Hunan and Heilongjiang provinces demonstrated that, even at the initial stage of resistance development, high resistance could occur in field conditions. At this stage of resistance development, does dimethachlon resistance in S. sclerotiorum develop faster in regions where dimethachlon is extensively applied than in regions with lower levels of application? There are still debates about the effects of fungicide application rate on the development of resistance. Some scientists believe that, compared with high rates of fungicide application, low rates may hasten the evolution of resistance by increasing mutation frequencies (11). Our results indicated that resistance emergence could not be inferred from the intensity of fungicide application, at least during the initial stage of the development of dimethachlon resistance in S. sclerotiorum.

Positive cross-resistance was observed among the dicarboximide fungicides dimethachlon, iprodione, and procymidone. This result

Table 3. Comparison of mycelial growth and sclerotial production on potato dextrose agar (PDA) plates between field-resistant and sensitive isolates of Sclerotinia sclerotiorum ${ }^{\mathrm{v}}$

\begin{tabular}{|c|c|c|c|c|c|}
\hline \multirow[b]{2}{*}{ Isolates $\left(\mathbf{E C}_{\mathbf{5 0}}\right)^{\mathrm{x}}$} & \multirow[b]{2}{*}{ Mycelial growth $(\mathrm{cm}) \pm \mathrm{SE}^{\mathrm{y}}$} & \multicolumn{2}{|c|}{ Sclerotia formation time (day) ${ }^{w}$} & \multirow[b]{2}{*}{ Number of sclerotia/dish } & \multirow[b]{2}{*}{ Sclerotia dry weight $(g)^{z}$} \\
\hline & & Start time & End time & & \\
\hline HLJMG (0.22) & $3.60 \pm 0.04 \mathrm{~d}$ & $5 \mathrm{a}$ & $8 \mathrm{a}$ & $19.5 \mathrm{~d}$ & $0.1144 \mathrm{c}$ \\
\hline GSMG $(0.23)$ & $2.80 \pm 0.20 \mathrm{c}$ & $5 \mathrm{a}$ & $8 \mathrm{a}$ & $16.0 \mathrm{c}$ & $0.1228 \mathrm{c}$ \\
\hline SXMG (0.38) & $3.30 \pm 0.18 \mathrm{~cd}$ & $5 \mathrm{a}$ & $8 \mathrm{a}$ & $12.8 \mathrm{~b}$ & $0.0519 \mathrm{~b}$ \\
\hline HLJ3 (25.32) & $1.70 \pm 0.05 \mathrm{a}$ & $11 \mathrm{c}$ & $15 \mathrm{c}$ & $10.3 \mathrm{a}$ & $0.0156 \mathrm{a}$ \\
\hline HLJ4 (28.10) & $1.90 \pm 0.09 \mathrm{ab}$ & $9 \mathrm{~b}$ & $12 \mathrm{~b}$ & $10.3 \mathrm{a}$ & $0.0839 \mathrm{~b}$ \\
\hline HN1 (31.27) & $2.10 \pm 0.14 \mathrm{~b}$ & $9 \mathrm{~b}$ & $12 \mathrm{~b}$ & $19.5 \mathrm{~d}$ & $0.0722 \mathrm{~b}$ \\
\hline
\end{tabular}

${ }^{v}$ A different letter in the same column indicates that the difference was significant according to Fisher's least significant difference test $(P=0.05)$ in SPSS

${ }^{w}$ Sclerotial production start time was the number of days from incubation to the initial formation of sclerotia. The end time was the number of days from incubation to the end of growth of sclerotia on a PDA plate.

${ }^{x}$ Half maximal $(50 \%)$ effective concentration $\left(\mathrm{EC}_{50}\right)$ values for dimethachlon. Isolates HLJMG, GSMG, and SXMG were dimethachlon-sensitive isolates collected from Heilongjiang, Gansu, and Shaanxi provinces, respectively. The dimethachlon-resistant isolates HLJ3 and HLJ4 were collected from Heilongjiang province and HN1 was collected from Hunan province.

${ }^{y} \mathrm{SE}=$ standard error.

${ }^{\mathrm{z}}$ Dry weight of sclerotia was the weight of all sclerotia in a PDA plate.

Table 4. Comparison of pathogenicity on detached leaves of oilseed rape plants between field-resistant and sensitive isolates of Sclerotinia sclerotiorum ${ }^{\mathrm{y}}$

\begin{tabular}{|c|c|c|c|c|c|c|}
\hline \multirow[b]{2}{*}{ Isolates $\left(\mathbf{E C}_{50}\right)^{\mathbf{z}}$} & \multicolumn{2}{|c|}{$24 \mathrm{~h}$} & \multicolumn{2}{|c|}{$48 \mathrm{~h}$} & \multicolumn{2}{|c|}{$72 \mathrm{~h}$} \\
\hline & $\mathrm{LD}(\mathrm{cm}) \pm \mathrm{SE}$ & DI (\%) & $\mathrm{LD}(\mathrm{cm}) \pm \mathrm{SE}$ & DI (\%) & $\mathrm{LD}(\mathrm{cm}) \pm \mathrm{SE}$ & DI (\%) \\
\hline GSMG (0.23) & $0.85 \pm 0.03 a$ & 50.0 & $2.14 \pm 0.09 c$ & 62.5 & $3.01 \pm 0.32 b$ & 75.0 \\
\hline SXMG (0.38) & $0.78 \pm 0.05 \mathrm{a}$ & 37.5 & $2.06 \pm 0.12 \mathrm{c}$ & 100.0 & $3.31 \pm 0.12 b$ & 100.0 \\
\hline HLJMG (0.22) & $0.94 \pm 0.06 \mathrm{a}$ & 87.5 & $2.34 \pm 0.16 \mathrm{c}$ & 100.0 & $3.52 \pm 0.17 b$ & 100.0 \\
\hline HLJ3 (25.32) & - & - & - & - & - & - \\
\hline HLJ4 (28.10) & - & - & $1.65 \pm 0.05 \mathrm{~b}$ & 12.5 & $1.38 \pm 0.40 \mathrm{a}$ & 37.5 \\
\hline HN1 (31.27) & - & - & $0.85 \pm 0.11 \mathrm{a}$ & 20.0 & $1.20 \pm 0.02 \mathrm{a}$ & 20.0 \\
\hline SX2 (29.57) & - & - & - & - & - & - \\
\hline
\end{tabular}

${ }^{\mathrm{y}} \mathrm{LD}=$ lesion diameter, $\mathrm{SE}=$ standard error, and $\mathrm{DI}=$ disease incidence (number of successful infections/number of total inoculations $\times 100$ ); - indicates no infection and, therefore, no data available. The same letter in the same column indicates that the difference was not significant according to Fisher's least significant difference test $(P=0.05)$ in SPSS.

${ }^{\mathrm{z}}$ Half maximal $(50 \%)$ effective concentration $\left(\mathrm{EC}_{50}\right)$ values. Isolates GSMG, SXMG, and HLJMG were dimethachlon-sensitive isolates collected from Gansu, Shaanxi, and Heilongjiang provinces, respectively. The dimethachlon-resistant isolates HLJ3 and HLJ4 were collected from Heilongjiang province, and HN1and SX2 were collected from Hunan province and Shaanxi province, respectively. 
was not surprising because these three fungicides have almost the same mode of action: acting on MAP/histidine kinase in osmotic signal transduction $(18,24)$. However, positive cross-resistance between the dicarboximide fungicides and diethofencarb was also detected. The mode of action of diethofencarb is the inhibition of $\beta$-tubulin assembly during mitosis (25). Positive cross-resistance between the two groups of fungicides with totally different modes of action could have been caused by a multi-drug-resistance mechanism such as the ATP-binding cassette, as reported in $B$. cinerea (16). Field isolates of $B$. cinerea resistant to both procymidone and diethofencarb have been reported but not all of the procymidoneresistant isolates were also resistant to diethofencarb (31), suggesting that procymidone resistance and diethofencarb resistance evolved independently in $B$. cinerea.

Previous studies showed that laboratory-induced dicarboximideresistant isolates of $B$. cinerea (6) and Alternaria brassicicola $(13,23)$ exhibited greater osmotic sensitivity and decreased virulence on hosts, whereas field-resistant isolates did not show increased osmotic sensitivity and retained high virulence on plants. In contrast, our results showed that the field-dimethachlon-resistant isolates of $S$. sclerotiorum were more sensitive to osmotic pressure, less virulent on the host plants oilseed rape, grew more slowly on PDA, and showed delayed sclerotia formation compared with the sensitive isolates. Colony characteristics such as mycelial radial growth rates of $S$. sclerotiorum in vitro have been related to pathogenicity under controlled environmental conditions $(8,40)$. However, studies on S. sclerotiorum isolates from Australia showed that there was no correlation between pigmentation or colony diameter on PDA with pathogenicity as measured by diameter of cotyledon lesions on the host Brassica napus (10). Genetic diversity studies on $S$. sclerotiorum demonstrated that genetic diversity was not related to the pathogenicity of the pathogen $(26,32)$. The present results indicated that the field-dimethachlon-resistant isolates had lower fitness to compete with the field-sensitive isolates under natural field conditions. This may have been one of the reasons for the slow development of dimethachlon resistance under field conditions and the low frequency of resistant isolates after many years of dimethachlon application. Due to the present resistance status, dimethachlon resistance in field isolates of $S$. sclerotiorum should be closely monitored in the next several years, and integrated pest management strategies such as biological, biotechnical, and cultural methods should be combined with chemical methods for controlling this devastating phytopathogen.

\section{Acknowledgments}

This study was supported by the Special Fund for Agro-scientific Research in the Public Interest (Number 201103016). We thank Prof. D. Jiang of Huazhong Agricultural University for kindly providing isolates of S. sclerotiorum and Prof. G. Schnabel of Clemson University for helpful suggestions regarding this manuscript.

\section{Literature Cited}

1. Abawi, G. S., and Grogan, R. G. 1979. Epidemiology of diseases caused by Sclerotinia species. Phytopathology 69:899-904.

2. Bardin, S. D., and Huang, H. C. 2001. Research on biology and control of Sclerotinia diseases in Canada. Can. J. Plant Pathol. 23:88-98.

3. Boland, G. J., and Hall, R. 1994. Index of plant hosts of Sclerotinia sclerotiorum. Can. J. Plant Pathol. 16:93-108.

4. Bolton, D. M., Thomma, B. P., and Nelson, B. D. 2006. Sclerotinia sclerotiorum (lib.) de Bray: Biology and molecular traits of a cosmopolitan pathogen. Mol. Plant Pathol. 7:1-16.

5. Bosch, F., Paveley, N., Shaw, M., Hobbelen, P., and Oliver, R. 2011. The dose rate debate: Does the risk of fungicide resistance increase or decrease with dose? Plant Pathol. 60:597-606.

6. Cui, W., Beever, R. E., Parkes, S. L., and Templeton, M. D. 2004. Evolution of an osmosensing histidine kinase in field strains of Botryotinia fuckeliana (Botrytis cinerea) in response to dicarboximide fungicide usage. Phytopathology 94:1129-1135.

7. Dai, F. M., Xu, T., Wolf, G. A., and He, Z. H. 2006. Physiological and molecular features of the pathosystem Arabidopsis thaliana L. and Sclerotinia sclerotiorum Libert. J. Integr. Plant Biol. 48:44-52.

8. Durman, S. B., Menendez, A. B., and Godeas, A. M. 2003. Mycelial compatibility groups in Buenos Aires field populations of Sclerotinia sclerotiorum (Sclerotiniaceae). Aust. J. Bot. 51:421-427.

9. Elmer, P. A. G., Gaunt, R. E., and Frampton, C. M. 1998. Spatial and tem- poral characteristics of dicarboximide-resistant strains of Monilinia fructicola and brown rot incidence in stone fruit. Plant Pathol. 47:530-536.

10. Garg, H., Kohn, L. M., Andrew, M., Li, H., Sivasithamparam, K., and Barbetti, M. J. 2010. Pathogenicity of morphologically different isolates of Sclerotinia sclerotiorum with Brassica napus and B. juncea genotypes. Eur. J. Plant Pathol. 126:305-315.

11. Gressel, J. 2011. Low pesticide rates may hasten the evolution of resistance by increasing mutation frequencies. Pest Manage. Sci. 67:253-257.

12. Hou, M. S., and Huang, J. B. 2006. Pages 146-147 in: Agricultural Plant Pathology. Science Press, Beijing.

13. Huang, R., and Levy, Y. 1995. Characterization of iprodione-resistant isolates of Alternaria brassicicola. Plant Dis. 79:828-833.

14. Hubbard, J. C., Subbarao, K. V., and Koike, S. T. 1997. Development and significance of dicarboximide resistance in Sclerotinia minor isolates from commercial lettuce fields in California. Plant Dis. 81:148-153.

15. Jo, Y. K., Niver, A. L., Rimelspach, J. W., and Boehm, M. J. 2006. Fungicide sensitivity of Sclerotinia homoeocarpa from golf courses in Ohio. Plant Dis. 90:807-813.

16. Kretschmer, M., Leroch, M., Mosbach, A., Walker, A.-S., Fillinger, S., Mernke, D., Schoonbeek, H.-J., Pradier, J.-M., Leroux, P., Waard, M. A. D., and Hahn, M. 2009. Fungicide-driven evolution and molecular basis of multidrug resistance in field populations of the grey mould fungus Botrytis cinerea. PLoS Pathog. 5:1-13.

17. Kuang, J., Wang, J. X., and Zhou, M. G. 2011. Monitoring carbendazim and dimethachlon resistance of Sclerotinia sclerotiorum obtained from the blight stems of rape in Jiangsu Province. Chin. Agric. Sci. Bull. 27:285-291.

18. Lan, B. D., Khor, H. Y., and Don, G. H. 2004. Dicarboximide resistance in field isolates of Alternaria alternate is mediated by a mutation in a twocomponent histidine kinase gene. Fungal Genet. Biol. 41:102-108.

19. Letham, D. B., Huett, D. O., and Trimboli, D. S. 1976. Biology and control of Sclerotinia sclerotiorum in cauliflower and tomato crops in coastal New South Wales. Plant Dis. 60:286-289.

20. Li, Y. G., and Chen, L. N. 2011. Preliminary exploration for growth characteristics and fungicide resistance of Sclerotinia sclerotiorum in soybean. Soybean Sci. 30:281-284.

21. Lu, G. 2003. Engineering Sclerotinia sclerotiorum resistance in oilseed crops. Afr. J. Biotechnol. 2:509-516.

22. Ma, H. X., Feng, X. J., Chen, Y., Chen, C. J., and Zhou, M. G. 2009. Occurrence and Characterization of dimethachlon insensitivity in Sclerotinia sclerotiorum in Jiangsu province of China. Plant Dis. 93:36-42.

23. Ma, Z., and Michailides, T. J. 2004. Characterization of iprodione-resistant Alternaria isolates from pistachio in California. Pestic. Biochem. Physiol. 80:75-84.

24. Ma, Z., Yan, L. Y., Luo Y., and Michailides, T. J. 2007. Sequence variation in the two-component histidine kinase gene of Botrytis cinerea associated with resistance to dicarboximide fungicides. Pestic. Biochem. Physiol. 88:300-306.

25. Malandrakis, A., Markoglou, A., and Ziogas, B. 2011. Molecular characterization of benzimidazole resistant $B$. cinerea field isolates with reduced or enhanced sensitivity to zoxamide and diethofencarb. Pestic. Biochem. Physiol. 99:118-124.

26. Malvarez, M., Carbone, I., Grunwald, N. J., Subbarao, K. V., Schafer, M., and Kohn, L. M. 2007. New populations of Sclerotinia sclerotiorum from lettuce in California and peas and lentils in Washington. Phytopathology 97:470-483

27. Pan, Y. L., Wang, Z. Y., and Wu, H. Z. 1997. Resistance to carbendazim and stability of the resistance in Sclerotinia sclerotiorum. Chin. J. Jiangsu Agric. Sci. 13:32-35.

28. Purdy, L. H. 1979. Sclerotinia sclerotiorum: History, disease, and symptomatology, host range, geographic distribution, and impact. Phytopathology 69:875-880.

29. Qi, Y. X., Chen, F. X., Su, X. Y., Ding K. J., Yu, J., and Jiang, M. S. 2006. Monitoring on carbendazim resistance of Sclerotinia sclerotiorum obtained from the blight stems of rape in Anhui Province. Chin. Agric. Sci. Bull. 22:371-373.

30. Shi, Z. Y., Zhou, M. G., Ye, Z. Y., Shi, J. Y., Chen, H. G., and Wang, Y. Z 2000. Resistance monitoring of Sclerotinia sclerotiorum to carbendazim. Chin. J. Jiangsu Agric. Sci. 16:226-229.

31. Sun, H. Y., Wang, H. C., Chen, Y., Li, H. X., Chen, C. J., and Zhou, M. G. 2010. Multiple resistance of Botrytis cinerea from vegetable crops to carbendazim, diethofencarb, procymidone, and pyrimethanil in China. Plant Dis. 94:551-556.

32. Sun, J. M., Irzykowski, W., Jedryczka, M., and Han, F. X. 2005. Analysis of the genetic structure of Sclerotinia sclerotiorum (Lib.) de Bary populations from different regions and host plants by random amplified polymorphic DNA markers. J. Integr. Plant Biol. 47:385-395.

33. Sylvester-Bradley, R., and Donald, C. M. 1984. A code for stages of development in oilseed rape (Brassica napus L.). Aspect. Appl. Biol. 6:399-419.

34. Wang, H. Z. 2007. Strategy for rapeseed industry development based on the analysis of rapeseed production and demand in China. Chin. J. Oil Crop Sci. 29:101-105.

35. Wang, J. X., Ma, H. X., Chen, Y., and Zhou, M. G. 2009. Sensitivity of Sclerotinia sclerotiorum from oilseed crops to boscalid in Jiangsu Province of China. Crop Prot. 28:882-886. 
36. Zhang, X. L., Sun, X. M., Zhang, G. F., Yi, H. J., and Qiang, Y. 2003. Preliminary report on the monitoring of the resistance of Sclerotinia sclerotiorum to carbendazim and its integrated management. Chin. J. Pestic. Sci. Adm. 24:18-22.

37. Zhang, X. L., Zhang, Z., Zhang, J. M., Zhang, G. F., and Sun, X. M. 1998. The reason and analysis for decrease of carbendazim efficacy against Sclerotinia sclerotiorum. Chin. J. Pestic. Sci. Adm. 19:15-17.
38. Zhou, B. W., and Luo, Q. 1994. Rapeseed Diseases and Control. China Commerce and Trade Press, Beijing.

39. Zhu, X. H., and Chen, X. L. 2010. The pathogenic and biological control of Sclerotinia sclerotiorum. Chin. J. Zhejiang Agric. Sci. 22:1035-1039.

40. Ziman, L., Jedryczka, M., and Srobarova, A. 1998. Relationship between morphological and biochemical characteristics of Sclerotinia sclerotiorum isolates and their aggressivity. J. Plant Dis. Prot. 105:283-288. 